$10-22-2020$

\title{
Superconductivity in single crystals of ZrP1.27Se0.73
}

K. W. Chen

G. Chappell

S. Zhang

W. Lan

Tiglet Besara

Missouri State University

See next page for additional authors

Follow this and additional works at: https://bearworks.missouristate.edu/articles-cnas

\section{Recommended Citation}

Chen, K-W., G. Chappell, S. Zhang, W. Lan, T. Besara, K. Huang, D. Graf, L. Balicas, A. P. Reyes, and R. E. Baumbach. "Superconductivity in single crystals of ZrP 1.27 Se 0.73." Physical Review B 102, no. 14 (2020): 144522.

This article or document was made available through BearWorks, the institutional repository of Missouri State University. The work contained in it may be protected by copyright and require permission of the copyright holder for reuse or redistribution.

For more information, please contact BearWorks@library.missouristate.edu. 


\section{Authors}

K. W. Chen, G. Chappell, S. Zhang, W. Lan, Tiglet Besara, K. Huang, D. Graf, L. Balicas, A. P. Reyes, and R. E. Baumbach 


\title{
Superconductivity in single crystals of $\mathrm{ZrP}_{1.27} \mathrm{Se}_{0.73}$
}

\author{
K.-W. Chen, ${ }^{1,2,3}$ G. Chappell, ${ }^{1,2}$ S. Zhang $\odot,{ }^{1,2}$ W. Lan $\odot,{ }^{1,2}$ T. Besara $\odot,{ }^{4}$ K. Huang, ${ }^{1}$ D. Graf, ${ }^{1}$ L. Balicas, ${ }^{1}$ A. P. Reyes $\odot,{ }^{1}$ \\ and R. E. Baumbach ${ }^{1}$ \\ ${ }^{1}$ National High Magnetic Field Laboratory, Florida State University, Tallahassee, Florida 32310, USA \\ ${ }^{2}$ Department of Physics, Florida State University, Tallahassee, Florida 32306, USA \\ ${ }^{3}$ Department of Physics, University of Michigan, Ann Arbor, Michigan 48109, USA \\ ${ }^{4}$ Physics, Astronomy, and Materials Science Department, Missouri State University, Springfield, Missouri 65897, USA
}

(Received 27 May 2020; revised 29 September 2020; accepted 2 October 2020; published 22 October 2020)

\begin{abstract}
Results are reported for single crystals of the PbFCl-type layered compound $\mathrm{ZrP}_{1.27} \mathrm{Se}_{0.73}$ that were produced using the iodine vapor transport method. Electrical transport, magnetization, and heat capacity measurements reveal disordered metallic behavior and the occurrence of bulk superconductivity, with a transition temperature $\left(T_{c}\right)$ of $7.1 \mathrm{~K}$ and an anisotropic orbitally limited upper critical field. ${ }^{31} \mathrm{P}$ nuclear magnetic resonance measurements provide additional microscopic information, where the line shape, Knight shift, and spin-lattice relaxation data are consistent with the superconductivity originating from the corrugated $\mathrm{Zr}-\mathrm{P}(2 \mathrm{c}) / \mathrm{Se}$ plane. This suggests that the superconductivity first forms in the corrugated plane and the bulk superconductivity eventually occurs via coupling between the square planar layers. These data also show that either there are multiple superconducting gaps or there is a single gap that does not fully open across the entire Fermi surface. These results clarify the superconducting state in this material and will enable further measurements that require single-crystal specimens.
\end{abstract}

DOI: 10.1103/PhysRevB.102.144522

\section{INTRODUCTION}

There is ongoing interest in the field of superconductivity, with efforts spanning from dense hydrogen systems with high transition temperatures [1] to unconventional Cooper pairing in $f$-electron systems [2] to potential topological superconductors [3]. In many cases, initial progress has been driven by surveys of families of materials, e.g., hydrogen sulfide [4], $\mathrm{UTe}_{2}$ [5], and chemically intercalated $\mathrm{Bi}_{2} \mathrm{Se}_{3}[6,7]$, where various crystal/chemical trends are often associated with their attractive properties. A particularly deep reservoir for superconductivity and other novel behaviors is systems that feature quasi-two-dimensional structural units, e.g., the $\mathrm{PbFCl}, \mathrm{ThCr}_{2} \mathrm{Si}_{2}$, and $\mathrm{CaBe}_{2} \mathrm{Ge}_{2}$ type structures [8]. Interest in these families of materials has produced recurring surges of effort, where an example is the discovery of high-temperature superconductivity in the Fe-(As,S,Se,Te) systems $[9,10]$.

This motivated work in the phosphide-chalcogenides $A_{1-x} B_{x} \mathrm{P}_{2-y} X_{y}(A=\mathrm{Zr}, \mathrm{Hf} ; B=\mathrm{Y}, \mathrm{Lu} ;$ and $X=\mathrm{S}, \mathrm{Se})$ [11-14], which crystallize in the tetragonal $\mathrm{PbFCl}$ structure and can be viewed as consisting of stacked corrugated $\mathrm{Zr}$ $\mathrm{P}(2 \mathrm{c}) /$ Se planes that are separated by $\mathrm{P}$ square nets. These materials are type II superconductors where the transition temperature varies with chemical substitution, and share some similarities with other quasi-two-dimensional superconductors such as $\mathrm{LaO}_{1-x} \mathrm{~F}_{x} \mathrm{BiS}_{2}[15,16]$. In addition, electronic structure calculations [12] suggest that there are linearly dispersing bands near the Fermi surface, similar to what is seen in the topological nodal fermion semimetal $\mathrm{ZrSiS}$ [17-19]. While it remains to be established that these bands are not merely conventional, this potentially places these materials at a focal point between superconductivity and topological protection. In order to fully clarify the behavior of these systems, it will be necessary to perform measurements on well-characterized single-crystal specimens to determine (i) the band structure and superconducting gap symmetry and (ii) whether the possible Dirac bands exhibit nontrivial topology.

Here we report on the electrical transport, magnetization, heat capacity, and ${ }^{31} \mathrm{P}$ nuclear magnetic resonance (NMR) for single crystals of $\mathrm{ZrP}_{1.27} \mathrm{Se}_{0.73}$ that were produced using the iodine vapor transport method. Bulk superconductivity emerges from disordered metallic behavior at $T_{c}=7.1 \mathrm{~K}$, which is larger than the value reported for polycrystalline [11] and earlier single-crystalline specimens [13]. Measurements of the upper critical field $H_{c 2}$ show a pronounced anisotropy between the $a b$ plane and the $c$ axis. We also report results for the electrical resistivity under hydrostatic pressure $(0 \leqslant$ $P \leqslant 1.7 \mathrm{GPa}$ ), where $T_{c}$ decreases with increasing $P$, similar to many other conventional superconductors [20] (see Supplemental Material [21]).

${ }^{31} \mathrm{P}$ NMR measurements additionally provide microscopic information about the superconducting state by showing that the two crystallographic sites associated with the $\mathrm{P}$ nets are separately identifiable. Importantly, the NMR signal whose line shape, Knight shift, and spin-lattice relaxation are consistent with superconductivity is found to originate from the corrugated $\mathrm{Zr}-\mathrm{P}(2 \mathrm{c}) / \mathrm{Se}$ plane. The relaxation data in the superconducting state suggests either a residual DOS in the superconducting gap or a multiple-gap structure due to anisotropy and strong hybridization of the electronic bands at the Fermi energy. While the NMR data provide strong 
evidence that superconductivity initially exists solely on the corrugated planes, we propose that bulk superconductivity eventually occurs at low enough temperatures via coupling between the square planar layers.

\section{EXPERIMENTAL METHODS}

Single crystals of $\mathrm{ZrP}_{1.27} \mathrm{Se}_{0.73}$ were grown using the chemical vapor transport method. A polycrystalline precursor was first prepared by solid-state reaction of the pure elements $\mathrm{Zr}: \mathrm{P}: \mathrm{Se}$ in the ratio of $2: 1: 2$ at $1000^{\circ} \mathrm{C}$ for 24 hours. The resulting powders were subsequently sealed under vacuum with $3 \mathrm{mg} / \mathrm{cm}^{3}$ of iodine in quartz tubes with lengths $l=$ $10 \mathrm{~cm}$ and diameters $d=18 \mathrm{~mm}$. The tubes were placed in a resistive tube furnace with one end at $800{ }^{\circ} \mathrm{C}$ (source) and the other at $900^{\circ} \mathrm{C}$ (drain) for 21 days. Following this reaction, platelike single crystals were produced with typical surface dimensions $a, b \approx 350 \times 350 \mu \mathrm{m}$ and thickness $t \approx 100 \mu \mathrm{m}$ [Fig. 1(a) inset]. The stoichiometry and crystalline structure were determined using energy-dispersive $\mathrm{x}$-ray spectroscopy and single-crystal x-ray diffraction measurements.

Magnetization $M$ measurements were performed on aligned single crystals of $\mathrm{ZrP}_{1.27} \mathrm{Se}_{0.73}$ using a Quantum Design magnetic properties measurement system. Electrical resistivity $\rho$ and heat capacity $C$ measurements were performed on aligned single crystals using a Quantum Design physical property measurement system for $1.8 \mathrm{~K} \leqslant T \leqslant$ 300 K. As shown in the Supplemental Material [21], similar behavior was observed throughout the batch, revealing that there is minimal chemical variation between crystals. The upper critical field $H_{c 2}$ was measured from $\rho(H)$ measurements at constant temperatures and sweeping field for $H<9 \mathrm{~T}$. The pressure $P$ dependence of $\rho(T)$ was measured using a pistoncylinder pressure cell (see Supplemental Material [21]) where the crystal was immersed in Daphne 7474 oil as the pressuretransmitting medium. $P$ was determined by measuring the superconducting transition of a slab of $6 \mathrm{~N}$ polycrystalline lead.

A $45 \mu \mathrm{g}$ single crystal was used for ${ }^{31} \mathrm{P}\left(I=1 / 2, \gamma_{n}=\right.$ 17.2356 MHz/T) NMR experiments. Despite the small sample size, measurements were possible because the ${ }^{31} \mathrm{P}$ isotope has $100 \%$ natural abundance and a relatively large gyromagnetic ratio $\left(\gamma_{n}\right)$ thereby giving a strong signal that makes it an ideal nuclear probe. The NMR spectra were obtained using a homemade NMR spectrometer with quadrature detection and fast Fourier transform of the Hahn echo. The typical $\pi / 2$ pulse is $\sim 1 \mu$ s which allows enough pulse bandwidth to cover the entire spectrum. The relaxation time was obtained using a single $90^{\circ}$ saturation pulse followed by a detection sequence. The resulting magnetization recovery was fitted with a single exponential appropriate for an $I=1 / 2$ system. A home-built NMR probe with a single-axis goniometer was used in a liquid ${ }^{4} \mathrm{He}$ flow cryostat. The temperature was monitored and regulated by LakeShore-340 temperature controller. A sorption-type ${ }^{3} \mathrm{He}$ refrigerator was used at temperatures below $1.5 \mathrm{~K}$.

\section{RESULTS AND DISCUSSION}

$\mathrm{ZrP}_{1.27} \mathrm{Se}_{0.73}$ exhibits disordered metallic behavior that culminates in bulk superconductivity below $T_{c}=7.1 \mathrm{~K}$, as

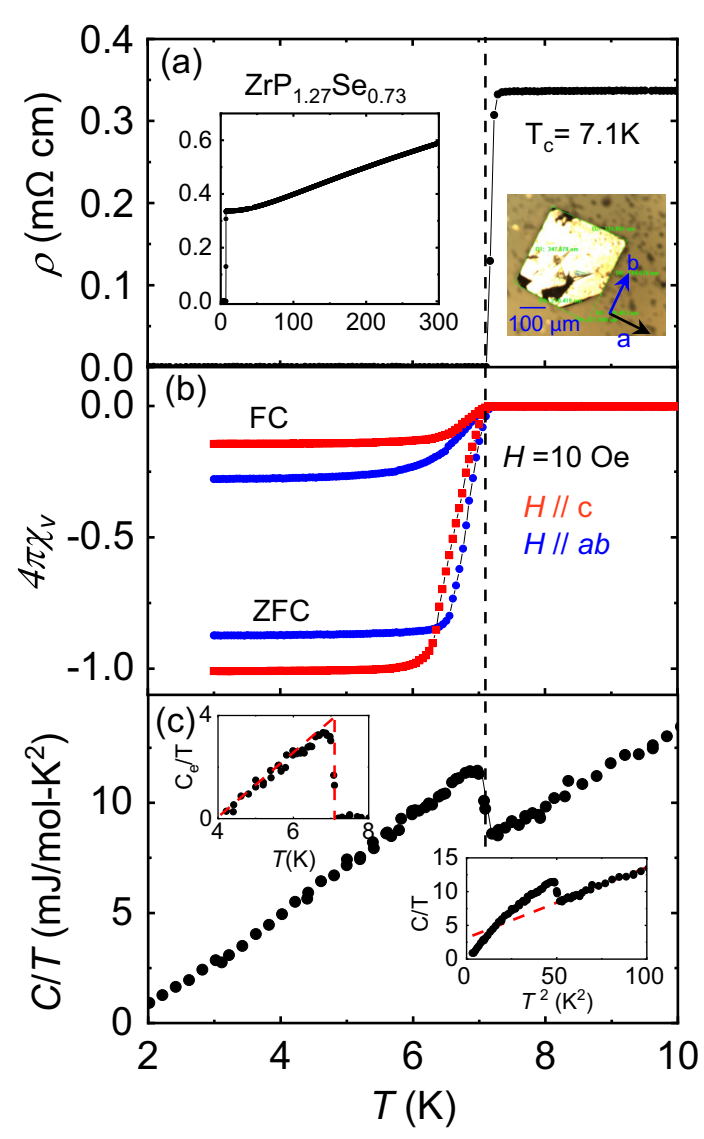

FIG. 1. (a) Electrical resistivity $\rho$ vs temperature $T$. The superconducting transition temperature $T_{c}=7.1 \mathrm{~K}$ is defined as the temperature where $\rho(T)$ reaches zero. The right inset shows a typical single-crystal specimen. (b) The zero-field-cooled (ZFC) and fieldcooled (FC) magnetic volume susceptibility under magnetic field $H=10 \mathrm{Oe}$. The ZFC susceptibility saturates to a maximum value of $-1(1 / 4 \pi)$ for $H \| c$ and $-0.9(1 / 4 \pi)$ for $H \| a b$. (c) Specific heat $C$ divided by $T$ vs $T$ for $1.8 \mathrm{~K} \leqslant T \leqslant 10 \mathrm{~K}$. The right inset shows $C / T$ vs $T^{2}$ where the dotted line is a fit to the data as described in the text. The left inset shows the electronic component of the heat capacity $C_{e} / T$. The dotted lines represent an equal entropy construction used to define $T_{c}$ and the size of the jump.

evidenced by electrical resistivity, magnetic susceptibility, and heat capacity measurements (Fig. 1). As shown in Fig. 1(a), the residual resistivity $\rho_{0} \approx 340 \mu \Omega / \mathrm{cm}$ indicates large disorder scattering for $T \gtrsim T_{c}$. This is consistent with earlier results from polycrystalline [11] and single-crystalline [13] samples which were grown by sintering under a pressure of $2 \mathrm{GPa}$, but we point out that here $T_{c}$ is noticeably larger than the previous maximum value ( $T_{c \max }=6.3 \mathrm{~K}$ at $\left.x=0.75\right)$. Taking into account the demagnetization factor [22] $d=0.67$ for $H \| c$ and $d=0.15$ for $H \| a b$, the zero-field-cooled (ZFC) and field-cooled (FC) magnetic volume susceptibility under a magnetic field $H=10$ Oe are shown in Fig. 1(b). The ZFC curve displays complete diamagnetic shielding $4 \pi \chi_{v} \approx-1$ for $H \| c$ and -0.9 for $H \| a b$; the FC curve shows $14 \%$ (27\%) of the full Meissner state for $H \| c(H \| a b)$ at $T=$ $2 \mathrm{~K}$. These suggest that the superconductivity is bulklike, but that the Meissner effect does not fully expel the magnetic 

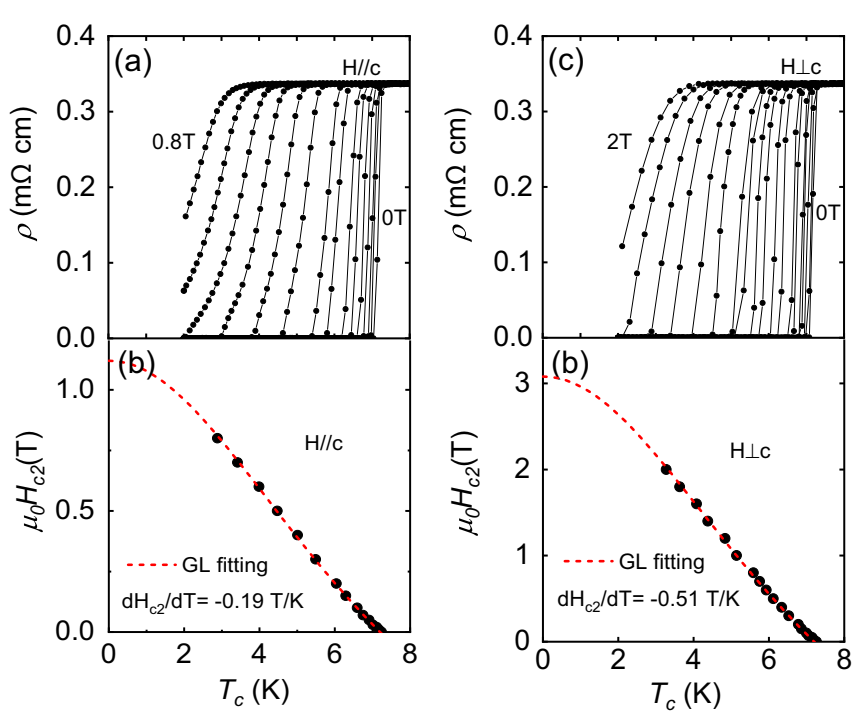

FIG. 2. Temperature dependence of the electrical resistivity $\rho(T)$ in various magnetic fields $H$ close to the superconducting transition. Panels (a) and (b) show $H$ applied parallel $\|$ and perpendicular $\perp$ to the $c$ axis, respectively. Panels (c) and (d) show the upper critical fields $H_{c 2}$ vs $T$ for $H \|$ and $\perp$ to the $c$ axis, respectively. The dashed lines are fits to the data using the Ginzburg-Landau theory.

field upon cooling through $T_{c}$ under an applied magnetic field, consistent with expectations for a type II superconductor.

That the superconductivity is bulklike is confirmed by heat capacity measurements [Fig. 1(c)], where a lambda anomaly is observed in $C / T$ at $T_{c}$. A linear fit to the data for $T>T_{c}$ using the function $C / T=\gamma+\beta T^{2}$ yields the Sommerfeld coefficient $\gamma \approx 3.1 \mathrm{~mJ} / \mathrm{mol} \mathrm{K}^{2}$ and $\beta=0.105 \mathrm{~mJ} / \mathrm{mol} \mathrm{K}^{4}$ [right inset of Fig. 1(c)]. From $\beta$ the Debye temperature $\theta_{D}=382 \mathrm{~K}$ is calculated. When the size of the jump in $C / T$ at $T_{c}$ is determined using the entropy-balance method [left inset of Fig. 1(c)], we find that $\Delta C / \gamma T_{c} \approx 1.15-1.3$, which is reduced from the expected value for a typical BCS superconductor in the weak electron-phonon coupling limit $\left(\Delta C / \gamma T_{c} \approx 1.43\right)$. The uncertainty in this value originates from ambiguity in defining the jump $\Delta C / T_{\mathrm{c}}$. Similar behavior has previously been observed for other BCS superconductors, including $\mathrm{MgB}_{2}$, where $\Delta C / \gamma T_{c} \approx 0.8-1.1$ [23,24].

The anisotropic suppression of $T_{c}$ for magnetic fields applied parallel to the $c$ axis and the $a b$ plane is summarized in Fig. 2. $T_{c}$ is defined as the temperature for $90 \% \rho_{n}$, where $\rho_{n}$ is the normal-state resistivity. From this, we find that $H_{c 2, c}<$ $H_{c 2, a, b}$. As shown in Figs. 2(b) and 2(d), the upper critical field curves are fitted using the Ginzburg-Landau expression [25], $\mu_{0} H_{c 2}(T)=\mu_{0} H_{c 2}(0) \times\left[1-\left(T / T_{c}\right)^{2}\right] /\left[1+\left(T / T_{c}\right)^{2}\right]$, from which it is possible to estimate the upper critical field $\mu_{0} H_{c 2}$. From this analysis, we find that $\mu_{0} H_{c 2}(0)=1.1 \mathrm{~T}$ for $H \| c$ and 3.1 T for $H \| a b$. Alternatively, the upper critical field for a single-band BCS-type superconductor is given by $H_{c 2}(T)=$ $-\left.A T_{c} \frac{d H_{c 2}}{d T}\right|_{\left(T=T_{c}\right)}$ where $A=0.69$ in the dirty limit [26]. For $H \| c(H \| a b)$, the slopes $\frac{d H_{c 2}}{d T}=-0.19 T / \mathrm{K}(-0.51 T / \mathrm{K})$ are determined for the temperature range $3 \mathrm{~K} \leqslant T \leqslant 6 \mathrm{~K}$. Taking $T_{c}=7.1 \mathrm{~K}$, the upper critical field can thus be estimated $0.94 \mathrm{~T}$ for $H \| c$ and $H_{c 2}=2.53 \mathrm{~T}$ for $H \| a b$. Note that following either method, these values for $H_{c 2}$ are larger

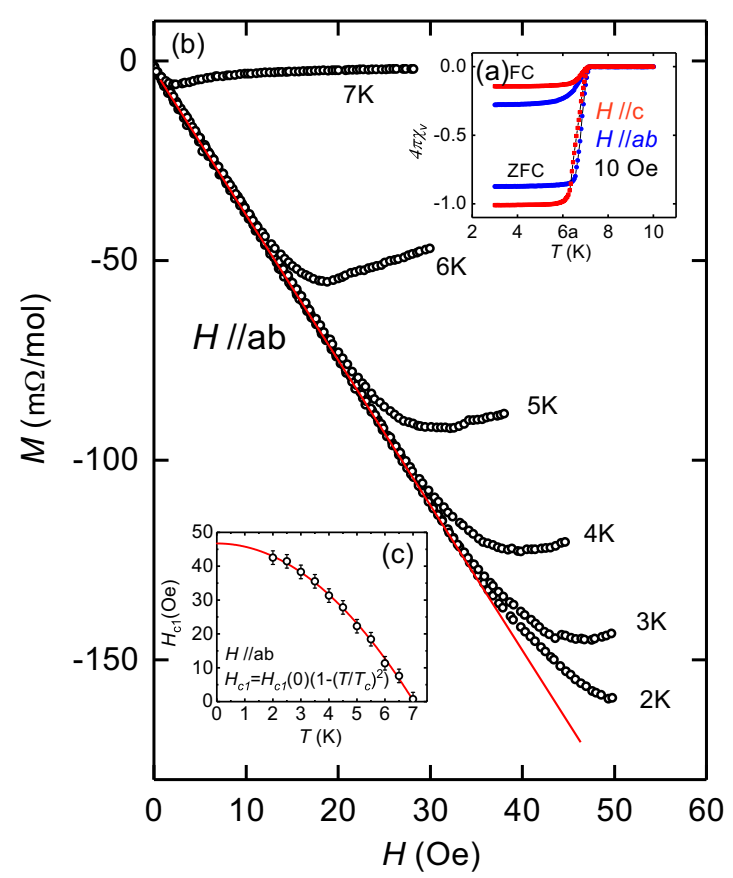

FIG. 3. (a) The zero-field-cooled (ZFC) and field-cooled (FC) magnetic volume susceptibility under magnetic field $H=10 \mathrm{Oe}$. The superconducting critical temperature $T_{c}$ is about $7.18 \mathrm{~K}$. The maximum ZFC susceptibility is $-0.9(1 / 4 \pi)$ for $H \| a b$ and $-1(1 / 4 \pi)$ for $H \| c$. (b) Magnetization $M$ vs magnetic field $H$ at various temperatures for $\mathrm{ZrP}_{1.27} \mathrm{Se}_{0.73}$. The black solid line is fit to the $2 \mathrm{~K}$ data in the low-field linear region. (c) Fitting $H_{c 1}$ data by the formula $H_{c 1}(T)=H_{c 1}(0)\left[1-\left(T / T_{c}\right)^{2}\right]$. The red solid line is the fitting curve.

than those previously reported for polycrystalline samples. It is also useful to compare to the Pauli limited upper critical fields $H_{c 2}=1.84 T_{c}=13.2 \mathrm{~T}$, which shows that the superconductivity is orbitally limited. The coherence length can also be calculated using the formula $H_{c 2}^{\| c}(0)=\Phi_{0} /\left[2 \pi \xi_{a b}^{2}(0)\right]$ and $H_{c 2}^{\| a b}(0)=\Phi_{0} /\left[2 \pi \xi_{a b}(0) \xi_{c}(0)\right]$, where $\Phi_{0}=h / 2 e$. The $\xi_{a b}(0)$ and $\xi_{c}(0)$ are calculated to be $17 \mathrm{~nm}$ and $6 \mathrm{~nm}$, respectively [25].

In Fig. 3 we show $M(H)$ at various $T$ where the behavior is consistent with expectations for a type II superconductor. Here, the black solid line is a linear fit to the low-field data, from which $H_{c 1}^{*}$ is defined as the field where $M(H)$ deviates from linear behavior. Taking into account the demagnetization factor and $H_{c 1}(T)=H_{c 1}^{*}(T) /(1-d)$, we extract the $H_{c 1}$ curve [Fig. 3(c)]. The zero-temperature lower critical field can be found by fitting the data to $\mu_{0} H_{c 1}(T)=\mu_{0} H_{c 1}(0) \times$ $\left[1-\left(T / T_{c}\right)^{2}\right]$ where $\mu_{0} H_{c 1}^{\| a b}=47$ Oe and $\mu_{0} H_{c 1}^{\| c}=124$ Oe. The in-plane superconducting penetration depth $\lambda_{a b}=$ $194 \mathrm{~nm}$ can be estimated by using the formula $\mu_{0} H_{c 1}^{\| c}=$ $\Phi_{0} /\left(4 \pi \lambda_{a b}^{2}\right)\left[\ln \kappa_{c}+0.5\right]$ where $\kappa_{c}=\lambda_{a b} / \xi_{a b}=11.4$. The interplane penetration depth $\lambda_{c}=745 \mathrm{~nm}$ can be estimated by using the formula $\mu_{0} H_{c 1}^{\| a b}=\Phi_{0} /\left(4 \pi \lambda_{a b} \lambda_{c}\right)\left[\ln \kappa_{a b}+0.5\right]$ where $\kappa_{a b}=\sqrt{\lambda_{a b} \lambda_{c}} / \sqrt{\xi_{a b} \xi_{c}}=55.8$.

In order to further inspect the microscopic details of the superconducting state, ${ }^{31} \mathrm{P}$ NMR measurements were performed. A typical ${ }^{31} \mathrm{P}$ NMR normal-state spectrum, col- 


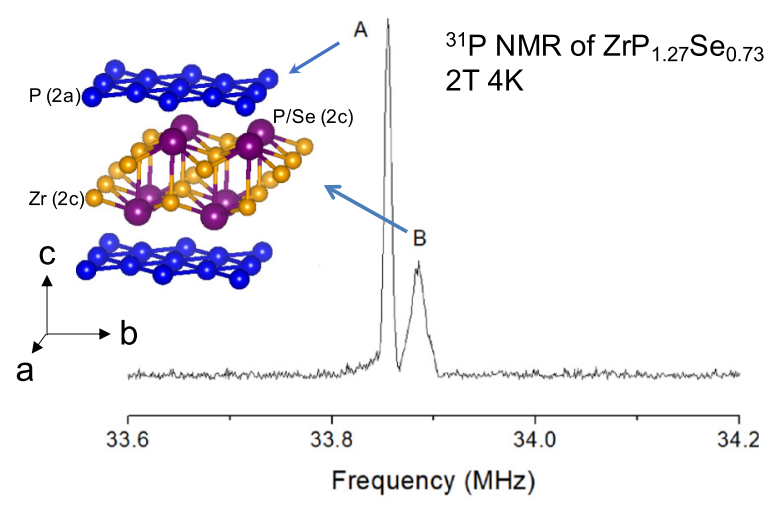

(a)

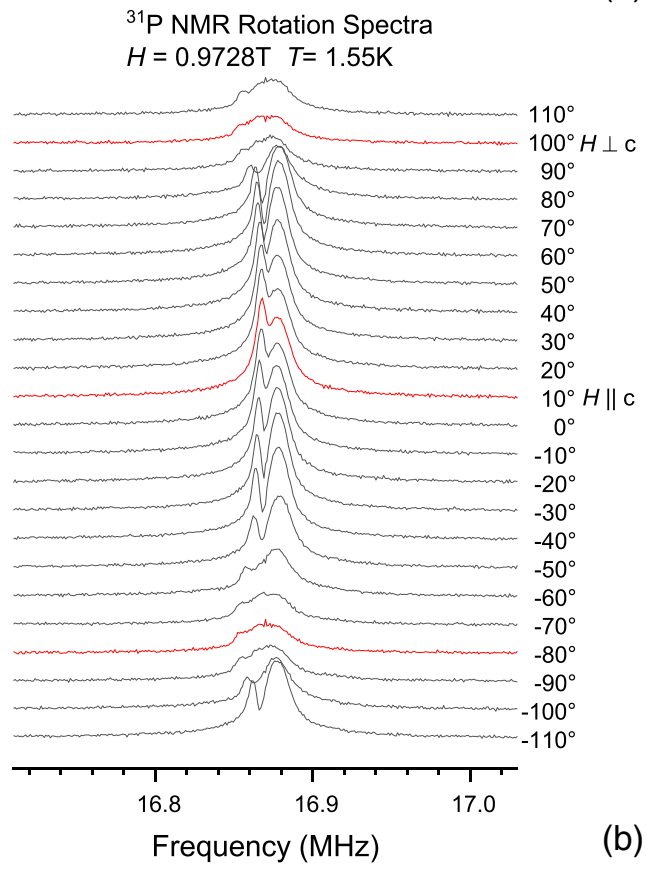

FIG. 4. (a) Crystal structure of $\mathrm{ZrP}_{2-x} \mathrm{Se}_{x}$. Typical ${ }^{31} \mathrm{P} \mathrm{NMR}$ spectra for $x=0.73$. The narrow line $\mathrm{A}$ is identified as originating from the square planar $\mathrm{P}(2 \mathrm{a})$ sites and the broad line $\mathrm{B}$ from the corrugated planar $\mathrm{P}(2 \mathrm{c})$ sites. (b) Angular dependence of ${ }^{31} \mathrm{P}$ spectra near $T_{c}$ and close to $H_{c 2}$. The left peak $\mathrm{A}$ is saturated in these spectra because of its long recovery time.

lected at $H=2 \mathrm{~T}$, is shown in Fig. 4(a). The spectrum consists of 2 peaks, where there is an intense and narrow line labeled "A" and a smaller and broader line "B" at higher frequency. At $4.2 \mathrm{~K}$, peak A exhibits a small frequency shift, $K_{A}=0.06 \%$, while peak B has about twice that value, $K_{B}=$ $0.11 \%$, with both being weakly temperature dependent. We assign the narrow $\mathrm{A}$ peak to the $\mathrm{P}$ atoms on the $\mathrm{P}(2 \mathrm{a})$ site and the $B$ peak to the $P(2 c)$ site. This assignment is made because a much more uniform electronic environment in the $\mathrm{P}(2 \mathrm{a})$ sites suggests a narrow line $(\mathrm{A})$ and the extra Se-injected electrons into the $\mathrm{P}(2 \mathrm{c})$ site would broaden the peak (B) and would contribute to additional shifts as well. For $x=0.73$, it is expected that the peak intensity ratio B:A is close to $1 / 3$ if all the Se atoms go to the $\mathrm{P}(2 \mathrm{c})$ sites, but due to the large difference in NMR relaxation between the two peaks, site assignment using signal intensities are not always reliable. Nevertheless, the contrasting spin dynamics demonstrated by the two peaks corroborates this site assignment. We observed that peak B has a very short spin-lattice relaxation $T_{1}$ (and a correspondingly short spin-coherence time, $T_{2}$ as well) which is more than an order of magnitude faster than peak A. Indeed, the short $T_{1}$ behavior is expected to occur in the carrier-rich corrugated layer $\mathrm{P}(2 \mathrm{c})$ as fluctuations from hybridized $\mathrm{Zr}_{d} / \mathrm{P}_{p}$ electrons on these layers create magnetic relaxation channels for the probe nuclei.

Figure 4(b) shows the spectral rotation pattern at $1.55 \mathrm{~K}$, close to transition temperature at low fields, $H^{*}=0.97 \mathrm{~T}$, which is above $H_{c 2, c}$ but below $H_{c 2, a b}$. At angles near $100^{\circ}$, peak B suddenly becomes broadened while peak A remains narrow. At the same time, peak B shifts toward lower frequencies. This demonstrates that the system enters the superconducting state as it is rotated in field. The B peak broadens due to magnetic vortices. The shift toward lower frequency is due to Cooper pairing which suppresses the spin part of the Knight shift. The data also show that superconductivity exists on the B site but not on the A site. This is reminiscent of planar (2D) superconductivity in high- $T_{c}$ cuprates. This observation is consistent with the relaxation data as shown below.

Figure 5 shows the temperature dependence of the spinlattice relaxation rates $1 / T_{1}$ for peaks $\mathrm{A}$ and $\mathrm{B}$ measured at $2 \mathrm{~T}$ field parallel to the planes. Both sites exhibit Korringa behavior above $T_{c}: T_{1} T(\mathrm{~A})=16(2) \mathrm{sK}$ and $T_{1} T(\mathrm{~B})=0.9(1) \mathrm{sK}$. As mentioned earlier, the one order of magnitude difference reflects the large electronic fluctuations occurring on the $\mathrm{P}(2 \mathrm{c})$ plane (B peak). Figure 6 shows the corresponding $1 / T_{1} T$ plots. Just below $T_{c}$, the slight bump, reminiscent of the coherence peak, is evident on this plot. It is then followed by a sudden downturn toward lower temperatures as would be expected from singlet pairing and formation of an energy gap. Similar behavior has been found in the ${ }^{75} \mathrm{As}$ NMR in structurally similar pnictide LiFeAs, but further work is needed to establish that there is a common origin for the behavior in both compounds [27].

In metals, the hyperfine interaction can be characterized by the Korringa relation [28], $\left(k^{2} T_{1} T\right)^{-1}=\alpha(0) k_{0}$, where $k_{0}^{-1}=\left(\gamma_{e} / \gamma_{n}\right)^{2} \hbar / 4 \pi k_{B}$ and the Stoner enhancement factor $\alpha(0)=1$ for noninteracting electron gas. For the ${ }^{31} \mathrm{P}$ isotope, $k_{0}^{-1}=1.6 \times 10^{-6} \mathrm{sK}$. Using the experimental data for site $\mathrm{B}$, we found that the ${ }^{31} \mathrm{P}$ relaxation is slightly enhanced, by a factor of $\alpha_{B}(0) \sim 1.5$. This enhancement is consistent with the strong hybridization among $\mathrm{P}(2 \mathrm{c})_{p}, \mathrm{Zr}_{d}$, and $\mathrm{Se}_{p}$ bands at the Fermi level. This result further justifies our site assignment. Note that for site A, the enhancement is very small, $\alpha_{A}(0) \sim 0.3$.

Comparing the relaxation rates of the two peaks as a function of temperature, we see in Fig. 5 a change in slope at $T_{c} \approx 3.7 \mathrm{~K}$ for the $\mathrm{B}$ site, while it remains Korringa (linear in $T$ ) for the A site. This suggests that superconductivity initially occurs on the $\mathrm{Zr}-\mathrm{P}(2 \mathrm{c}) / \mathrm{Se}$ layer, a conclusion consistent with the rotation data. Band structure calculation and doping dependence suggest that the main conduction layer is on this plane which leads to an increase in the density of states (DOS) when Se is doped into the system [12]. 

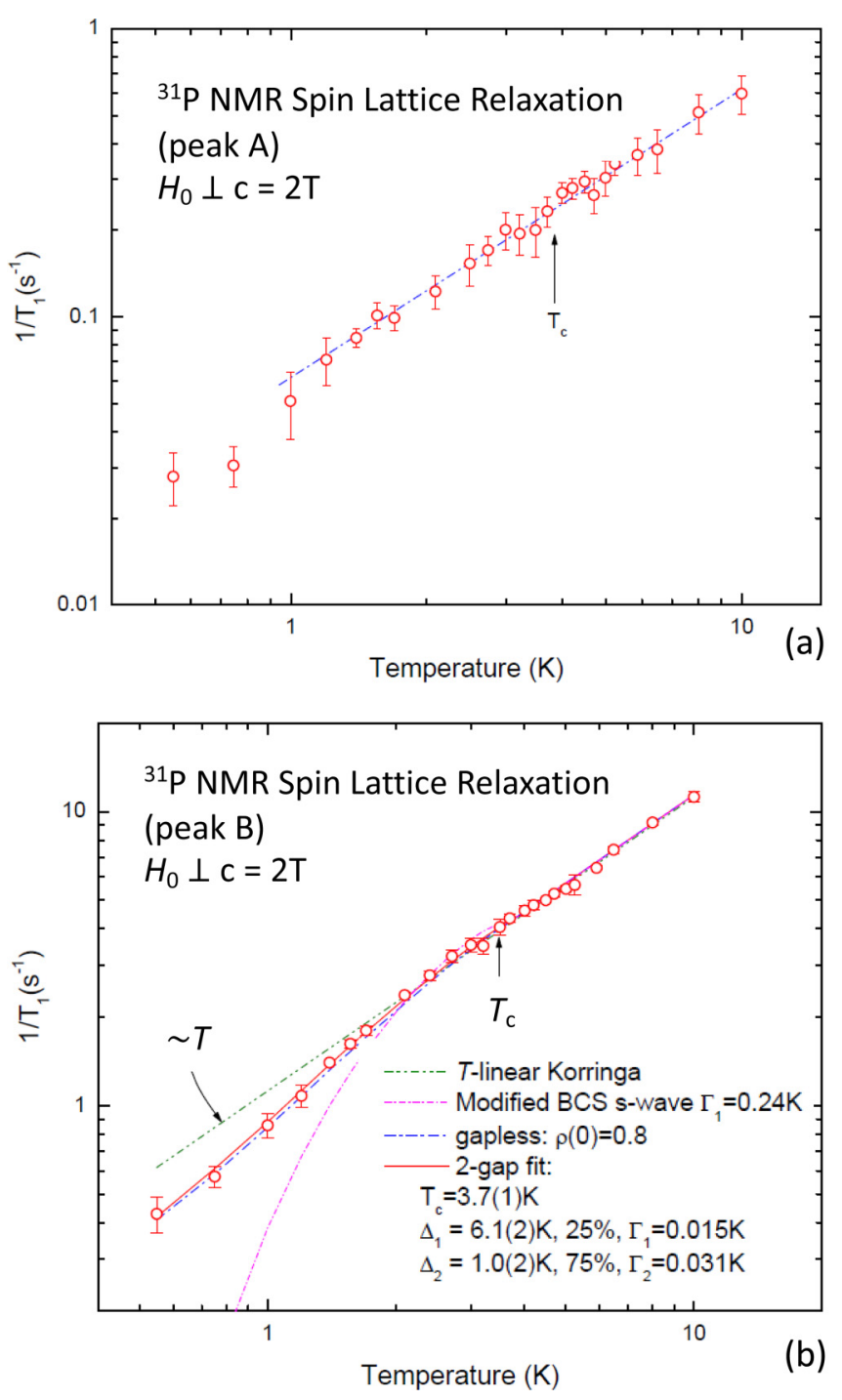

FIG. 5. (a) Temperature dependence of ${ }^{31} \mathrm{P}$ spin-lattice relaxation in the $\mathrm{P}(2 \mathrm{a})$ site (peak A) and (b) $\mathrm{P}(2 \mathrm{c})$ site (peak B). For (b), the data are fitted with various modified BCS functions.

The NMR relaxation rate $1 / T_{1}$ is related to the density of states $\rho(E, T)$ as follows:

$$
\frac{1}{T_{1}} \propto \int_{-\infty}^{\infty}|\rho(E, T)|^{2} f(E)[1-f(E)] d E,
$$

where $f(E)$ is the Fermi-Dirac distribution function. For a weak-coupling BCS superconductor the opening of a gap at the Fermi energy below $T_{c}$ results in a spike of DOS on the band edge. Concomitantly, the relaxation rate is enhanced, a phenomenon known as the Hebel-Slichter peak or coherence peak. The absence of the coherence peak suggests that the Fermi energy is not fully gapped and the bands are strongly hybridized around FS. For a simple band BCS model, the density of states $\rho(E, T)$ in the superconducting state may be modeled as

$$
\rho(E, T)=\operatorname{Re}\left(\frac{E-i \Gamma}{\sqrt{(E-i \Gamma)^{2}-\Delta(T)^{2}}}\right)+\rho_{0},
$$
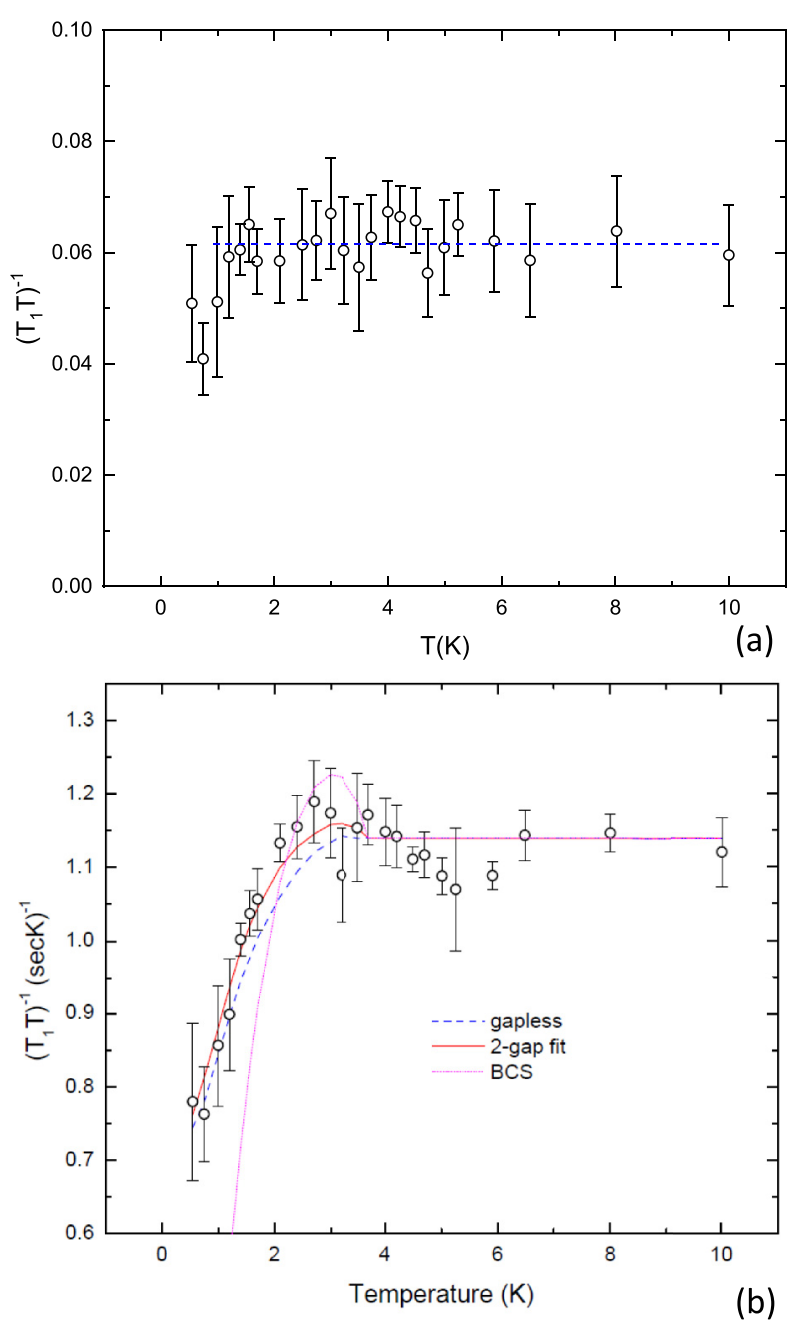

FIG. 6. (a) ${ }^{31} \mathrm{P}$ spin-lattice relaxation rate divided by temperature in the $\mathrm{P}(2 \mathrm{a})$ site (peak A) and (b) for the $\mathrm{P}(2 \mathrm{c})$ site (peak B) for $H \perp c$. The loss in density of states below $T_{c}$ is clearly seen in peak $\mathrm{B}$ but not in A. The dashed line in (a) is a guide to the eye. Lines in (b) are the same fit as in Fig. 5.

where $\rho_{0}$ is the residual DOS and $\Delta(T)$ is the BCS order parameter. Due to the finite-lifetime effects of the quasiparticles on the gap edge, the thermal smearing factor $\Gamma$ is utilized in our calculations to smoothen the singularities in the DOS. Figures 5(b) and 6(b) show fits to the relaxation curve using the above model as a basis. We note that a simple BCS gap fails to fit the data but a model in which there are residual states in the gap fits the data well. In order to explain the doping dependence of $T_{c}$, it was earlier suggested [12] that either an anisotropic gap or multiple gaps may exist. We fitted the data to a two-gap model as shown. We obtained $6.1 \mathrm{~K}$ and $1.0 \mathrm{~K}$ for the large and small gaps, respectively, with $75 \%$ of the weight on the small gap. The model fits the data much better, especially at lower temperatures than the fit using a finite residual DOS. However, the resolution of data does not permit us to confidently discard one or the other. In any case, it is clear that the low-temperature $1 / T_{1}$ behavior is nonexponential which suggests that the superconducting gap does not fully open in this material. It is worth noting that $\mathrm{Fe}$ pnictides show a similar power-law behavior which is claimed 
to be from the strong-coupling effects and impurity scattering $[29,30]$.

\section{CONCLUSION}

Single crystals of the compound $\mathrm{ZrP}_{1.27} \mathrm{Se}_{0.73}$ prepared by the chemical vapor pressure technique exhibit a superconducting transition near $7.1 \mathrm{~K}$ that has been confirmed in resistivity, magnetic susceptibility, and heat capacity measurements. The transition temperature $T_{c}$ is suppressed anisotropically for magnetic fields perpendicular and parallel to the $c$ axis. It is also seen that $T_{c}$ decreases as pressure increases, which might suggest that the negative pressure could lead to larger $T_{c}$, e.g., in $\mathrm{ZrAs}_{2-x} \mathrm{Se}_{x}, \mathrm{HfAs}_{2-x} \mathrm{Se}_{x}$, or $\mathrm{HfAs}_{2-x} \mathrm{Te}_{x}$. However, prior results for $\mathrm{ZrAs}_{2-x} \mathrm{Se}_{x}$ show that it is not a superconductor and that the $T_{c}$ of $\mathrm{HfAs}_{1.7} \mathrm{Se}_{0.2}$ and $\mathrm{HfAs}_{1.67} \mathrm{Te}_{0.12}$ are found to be only $0.52 \mathrm{~K}$ and $1.67 \mathrm{~K}$, respectively $[14,31,32]$.

${ }^{31} \mathrm{P}$ NMR measurements provide microscopic information about the nature of superconductivity in this system. The two crystallographic sites have been separately identified in the NMR spectra. The NMR line whose line shape, Knight shift, and spin-lattice relaxation exhibit behavior consistent with superconductivity is identified to originate from the corrugated $\mathrm{Zr}-\mathrm{P}(2 \mathrm{c}) / \mathrm{Se}$ plane. The relaxation data in the superconducting state suggest either a residual DOS in the superconducting gap or a multiple-gap structure due to anisotropy and strong hybridization of the electronic bands at the Fermi energy. The NMR data provide strong evidence that superconductivity initially exists solely on these planes, and it is possible that bulk superconductivity eventually occurs via tunneling mechanism through the square planar layers. Taken together, these results clarify the superconductivity of this material and will aid in further investigations of this developing family of materials.

\section{ACKNOWLEDGMENTS}

This work was performed at the National High Magnetic Field Laboratory (NHMFL), which is supported by National Science Foundation Cooperative Agreement No. DMR1157490, the State of Florida, and the DOE. A portion of this work was supported by the NHMFL User Collaboration Grant Program. R.B. acknowledges support from the National Science Foundation through NSF/DMR1904361. L.B. is supported by the US Department of Energy Basic Energy Sciences program through Award No. DE-SC0002613.
[1] C. J. Pickard, I. Errea, and M. I. Eremets, Annu. Rev. Condens. Matter Phys. 11, 57 (2019).

[2] C. Pfleiderer, Rev. Mod. Phys. 81, 1551 (2009).

[3] L. Fu and E. Berg, Phys. Rev. Lett. 105, 097001 (2010).

[4] A. P. Drozdov, M. I. Eremets, I. A. Troyan, V. Ksenofontov, and S. I. Shylin, Nature (London) 525, 73 (2015).

[5] S. Ran, C. Eckberg, Q.-P. Ding, Y. Furukawa, T. Metz, S. R. Saha, I.-L. Liu, M. Zic, H. Kim, J. Paglione, and N. P. Butch, Science 365, 684 (2019).

[6] Y. S. Hor, A. J. Williams, J. G. Checkelsky, P. Roushan, J. Seo, Q. Xu, H. W. Zandbergen, A. Yazdani, N. P. Ong, and R. J. Cava, Phys. Rev. Lett. 104, 057001 (2010).

[7] S. Sasaki, M. Kriener, K. Segawa, K. Yada, Y. Tanaka, M. Sato, and Y. Ando, Phys. Rev. Lett. 107, 217001 (2011).

[8] M. Shatruk, J. Solid State Chem. 272, 198 (2019).

[9] G. R. Stewart, Rev. Mod. Phys. 83, 1589 (2011).

[10] D. C. Johnston, Adv. Phys. 59, 803 (2010).

[11] H. Kitô, Y. Yanagi, S. Ishida, K. Oka, Y. Gotoh, H. Fujihisa, Y. Yoshida, A. Iyo, and H. Eisaki, J. Phys. Soc. Jpn. 83, 074713 (2014).

[12] S. Ishida, H. Fujihisa, I. Hase, Y. Yanagi, K. Kawashima, K. Oka, Y. Gotoh, Y. Yoshida, A. Iyo, H. Eisaki, and H. Kitô, Supercond. Sci. Technol. 29, 055004 (2016).

[13] H. Kitô, K. Iwakiri, T. Nishio, K. Kawashima, S. Ishida, K. Oka, H. Fujihisa, Y. Gotoh, A. Iyo, H. Ogino, H. Eisaki, and Y. Yoshida, J. Phys.: Conf. Ser. 1054, 012003 (2018).

[14] J. Peng, J. Yu, S. Zhang, and G. Chen, Supercond. Sci. Technol. 31, 015020 (2018).

[15] Y. Mizuguchi, S. Demura, K. Deguchi, Y. Takano, H. Fujihisa, Y. Gotoh, H. Izawa, and O. Miura, J. Phys. Soc. Jpn. 81, 114725 (2012).
[16] D. Yazici, K. Huang, B. D. White, A. H. Chang, A. J. Friedman, and M. B. Maple, Philos. Mag. 93, 673 (2013).

[17] L. M. Schoop, M. N. Ali, C. Straber, A. Topp, A. Varykhalov, D. Marchenko, V. Duppel, S. S. P. Parkin, B. V. Lotsch, and C. R. Ast, Nat. Commun. 7, 11696 (2016).

[18] S. Pezzini, M. R. van Delft, L. M. Schoop, B. V. Lotsch, A. Carrington, M. I. Katsnelson, N. E. Hussey, and S. Wiedmann, Nat. Phys. 14, 178 (2018).

[19] M. N. Ali, Leslie M. Schoop, C. Garg, J. M. Lippmann, E. Lara, B. Lotsch, and S. S. P. Parkin, Sci. Adv. 2, e1601742 (2016).

[20] A. Eiling and J. S. Schilling, J. Phys. F 11, 623 (1981).

[21] See Supplemental Material at http://link.aps.org/supplemental/ 10.1103/PhysRevB.102.144522 for summary of (i) temperature-dependent magnetization and heat capacity of multiple different single-crystal specimens and (ii) pressure dependence of the superconducting transition temperature that was extracted from temperature-dependent electrical resistivity measurements.

[22] A. Aharoni, J. Appl. Phys. 83, 3432 (1998).

[23] Y. Wang, T. Plackowski, and A. Junod, Physica C 355, 179 (2001).

[24] H. D. Yang, J.-Y. Lin, H. H. Li, F. H. Hsu, C. J. Liu, S.-C. Li, R.-C. Yu, and C.-Q. Jin, Phys. Rev. Lett. 87, 167003 (2001).

[25] V. L. Ginzburg and L. D. Landau, Zh. Eksp. Teor. Fiz. 20, 1064 (1950).

[26] N. R. Werthamer, E. Helfand, and P. C. Hohenberg, Phys. Rev. 147, 295 (1966).

[27] S.-H. Baek, L. Harnagea, S. Wurmehl, B. Büchner, and H.-J. Grafe, Eur. Phys. J. B 85, 159 (2012).

[28] A. Narath and H. T. Weaver, Phys. Rev. 175, 373 (1968).

[29] A. V. Chubukov, D. V. Efremov, and I. Eremin, Phys. Rev. B 78, 134512 (2008). 
[30] D. Parker, O. V. Dolgov, M. M. Korshunov, A. A. Golubov, and I. I. Mazin, Phys. Rev. B 78, 134524 (2008).

[31] T. Cichorek, L. Bochenek, M. Schmidt, A. Czulucki, G. Auffermann, R. Kniep, R. Niewa, F. Steglich, and S. Kirchner, Phys. Rev. Lett. 117, 106601 (2016).
[32] A. Czulucki, G. Auffermann, M. Bednarski, L. Bochenek, M. Bohme, T. Cichorek, R. Niewa, N. Oeschler, M. Schmidt, F. Steglich, and R. Kniep, ChemPhysChem 11, 2639 (2010). 